\title{
COMPETÊNCIA TÉCNICA NA PREVENÇÃO DO HIVIAIDS: VALIDAÇÃO DE UM INSTRUMENTO
}

Gilson de Vasconcelos Torres ${ }^{1}$

Márcia Caron Ruffino ${ }^{2}$

Torres GV, Ruffino MC. Competência técnica na prevenção do HIVIAIDS: validação de um instrumento. Rev Latino-am Enfermagem 2001 novembro-dezembro; 9(6):7-12.

O estudo teve como objetivo construir e validar um instrumento para mensurar a competência técnica das ações educativas desenvolvidas pelo enfermeiro, na prevenção do HIVIAIDS nas unidades básicas de saúde (UBS). As etapas percorridas para a construção e testagem da fidedignidade do instrumento, foram: elaboração de itens relacionados a competência técnica na prevenção do HIV/AIDS; aplicação do instrumento numa amostra de 30 enfermeiros de diferentes instituições hospitalares e de ensino. 0 instrumento apresentou um nível satisfatório de estabilidade, homogeneidade e reprodutibilidade.

DESCRITORES: HIV, AIDS, enfermagem

\section{TECHNICAL COMPETENCE IN THE PREVENTION OF HIVIAIDS: VALIDATION OF AN INSTRUMENT}

This study aimed at building and validating an instrument to measure the technical competence of educational actions developed by nurses in the prevention of HIVIAIDS in Basic Health Units (BHU). The phases for constructing and testing the fidelity of the instrument were: elaboration of items related to the technical competence in the prevention of HIVIAIDS; application of the instrument in a sample of 30 male nurses from different hospitals and teaching institutions. The instrument presented a satisfactory level of stability, homogeneity and reproduction.

KEY WORDS: HIV, AIDS, nursing

\section{COMPETENCIA TÉCNICA EN LA PREVENCIÓN DE HIVIAIDS: VALIDACIÓN DE UN INSTRUMENTO}

El estudio tuvo como objetivo construir y validar un instrumento para medir la competencia de las acciones educativas desarrolladas por el enfermero en la prevención de HIVIAIDS en las unidades básicas de salud (UBS). Las etapas recorridas para la construcción y prueba de fidedignidad del instrumento, fueron: elaboración de ítems relacionados con la competencia técnica en la prevención de HIVIAIDS; aplicación del instrumento en una muestra de 30 enfermeros de diferentes instituciones hospitalarias y de enseñanza. El instrumento presentó un nivel satisfactorio de estabilidad, homogeneidad y reproductibilidad.

DESCRIPTORES:VIH, SIDA, enfermería

${ }^{1}$ Professor Adjunto da Universidade Federal do Rio Grande do Norte, Doutor em Enfermagem Fundamental, e-mail: gvt@ufrnet.br; ${ }^{2}$ Professor Titular. Escola de Enfermagem de Ribeirão Preto da Universidade de São Paulo, Centro Colaborador da OMS para o desenvolvimento da pesquisa em enfermagem 


\section{INTRODUÇÃO}

A evolução da disseminação da AIDS vem mostrando que a transmissão do HIV faz-se, primordialmente, por comportamentos de risco relacionados a práticas sexuais e uso de drogas injetáveis, sendo o principal modo de transmissão do HIV a conduta sexual, podendo atingir qualquer indivíduo, independente de características pessoais ou sociais.

Essa questão de mudança de hábitos, particularmente nos campos diretamente implicados na transmissão do HIV, está bem longe de ser simples e transcende, sem dúvida, o aspecto informativo, demanda o constante aprofundamento do debate e da reflexão em torno de práticas educativas, de modo a aumentar sua eficácia e apontar caminhos que respondam aos diversos desafios a elas relacionadas. Dessa forma, a eficácia da educação dependerá do nível de abrangência e contextualização com que for planejada e desenvolvida.

Todavia, para que o enfermeiro possa orientar seus clientes/ comunidade, necessita possuir conhecimentos e habilidades técnicas, pedagógicas e de planejamento sobre as práticas educativas em HIVIAIDS, a fim de desenvolver e administrar tal processo educativo.

A preocupação com a atuação do enfermeiro na prevenção do HIVIAIDS nas UBS, motivou-nos a estudar a competência técnica desse profissional no desenvolvimento das atividades educativas no combate a disseminação desta enfermidade ${ }^{(1)}$. Portanto, 0 objetivo deste estudo foi construir e testar a fidedignidade do instrumento para mensurar o nível de competência técnica do enfermeiro na prevenção do HIVIAIDS, nas UBS.

Competência está sendo entendida como capacidade de encontrar problemas e de resolvê-los, fazer determinada coisa, ter habilidade e aptidão. A competência a ser estudada refere-se ao conjunto de conhecimentos do enfermeiro no tocante aos aspectos relativos ao HIVIAIDS e ações de prevenção, no âmbito das UBS.

A validade e a fidedignidade são medidas que refletem a qualidade do instrumento, as quais interferem na credibilidade dos resultados da pesquisa em sua utilidade prática. Estudos dessa natureza, visando o desenvolvimento de estratégias de coletas de dados com evidências garantidas de validade e fidedignidade, são escassos na literatura de enfermagem ${ }^{(2-4)}$.

\section{MÉTODOS}

Trata-se de um estudo descritivo, realizado em Natal/RN, com enfermeiros que trabalham em quatro instituições públicas ligadas ao ensino e à assistência hospitalar e ambulatorial.
Construção do instrumento

Elaborado a partir dos referenciais do Ministério da Saúde ${ }^{(5-}$

${ }^{7)}$, e está constituído por duas partes: caracterização dos sujeitos e questões sobre a competência técnica em HIVIAids e sua prevenção.

Procedimento da validação aparente e de conteúdo do instrumento

Sabemos que uma amostra, verdadeiramente representativa de ítens, é muito difícil. Entretanto, procuramos minimizar a dificuldade, fazendo uma análise cuidadosa dos conteúdos, observando a importância e a determinação dos mesmos, de forma a contemplar os aspectos referentes à competência técnica que desejamos mensurar.

Submetemos 0 instrumento à validação aparente e de conteúdo aplicando-o a cinco (5) juizes, docentes de graduação em enfermagem da Universidade Federal do Rio Grande do Norte (UFRN)

0 instrumento foi apresentado pelo pesquisador que solicitou aos juizes analisarem e darem sugestões quanto à clareza das afirmações, facilidade de leitura e compreensão, forma de apresentação do instrumento, abrangência e representatividade do conteúdo contido nas questões sobre as competências técnicas do enfermeiro nas ações educativas na prevenção do HIVIAIDS e demais partes do instrumento. Solicitou ainda, que analisassem o conteúdo de cada afirmativa constante na parte II do instrumento, referente à competência técnica em HIVIAIDS, quanto ao seu poder discriminativo. $O$ julgamento deveria ser expresso com a letra $\mathrm{C}$ se concordasse com a afirmativa e $\mathrm{D}$ se discordasse das mesmas.

Modificamos 0 instrumento, acatando as criticas e sugestões quanto à clareza das afirmações, facilidade de leitura, compreensão e forma de apresentação do instrumento. $O$ instrumento foi considerado por todos os juizes abrangente e o conteúdo representativo para mensuração da competência técnica do enfermeiro na prevenção do HIVIAIDS.

Estabelecemos como critério de aceitação dos ítens referentes à competência técnica, parte II do instrumento, aqueles com poder discriminativo - Concordância ou Discordância - igual ou superior a $80 \%$ de concordância entre os juizes, conforme preconizado por estatístico ${ }^{(8)}$. Com base na apreciação dos juizes, todos os 30 itens, contidos na parte II, obtiveram concordância superior ao estabelecido para aceitação. Após as sugestões e modificações apontadas pelos juizes nas validações, aparente e de conteúdo, o instrumento foi submetido a testagem.

Testagem do instrumento

Para o teste do instrumento foi utilizada a amostragem 
aleatória simples com trinta (30) enfermeiros de quatro instituições públicas, sendo uma de ensino, uma UBS e duas hospitalares, tendose como critérios de inclusão: ter realizado algum treinamento e/ou capacitação em HIVIAIDS (Grupo A); não ter realizado nenhum treinamento e/ou capacitação em HIVIAIDS (Grupo B) e aceitar participar voluntariamente do teste para ambos os grupos.

0 grupo dos enfermeiros treinados e/ou capacitados em HIVIAIDS foi chamado de (Grupo A) e o grupo de enfermeiros não treinados em HIVIAIDS constitui (Grupo B). A comparação entre esses dois grupos teve a finalidade de observar a variabilidade de escores na parte referente à competência técnica em HIVIAIDS da parte II do instrumento entre os pesquisados.

\section{RESULTADOS E DISCUSSÃO}

Os participantes da testagem eram do gênero feminino, com faixa etária predominante de 31 a 45 anos $(83,4 \%)$, sendo que 53,3\% tinham licenciatura em enfermagem e 63,3\% especialização.

No tocante aos cursos e treinamentos em HIVIAIDS freqüentados pelos sujeitos, a capacitação técnica para enfermeiros em HIVIAIDS (73,3\%), aconselhamento em DST/HIVIAIDS (46,7\%) e DST e AIDS com 20,0\% foram os mais citados.

Como podemos observar na Tabela 1, em ambos os grupos, os participantes responderam em sua maioria, que se consideravam competentes em parte para atuar na prevenção do HIVIAIDS e apenas $26,7 \%$ dos profissionais treinados afirmaram tal competência.

Tabela 1 - Distribuição dos pesquisados segundo as respostas sobre a sua competência para atuar na prevenção do HIVIAIDS. Natal/RN, 1999

GRUPOS

\begin{tabular}{ccc} 
RESPOSTA & $\begin{array}{c}\text { A (treinados) } \\
\%\end{array}$ & $\begin{array}{c}\text { B (nẫo treinados) } \\
\%\end{array}$ \\
\hline Sim & 26,7 & 6,7 \\
Nẫo & 13,3 & 40,0 \\
Em parte & 60,0 & 53,3 \\
\hline
\end{tabular}

Para verificar o nível de consistência (fidedignidade) da parte II do instrumento, utilizamos o Índice Kappa (K), que é um indicador de concordância ajustado, pois leva em consideração, descontando no cômputo final, a concordância devida ao fator chance. Esse índice informa a proporção de concordância além da esperada pela chance e varia de "menos 1" a "mais 1", ou seja, quanto mais próximo de um (1) melhor o nível de concordância entre os observadores $^{(9)}$.

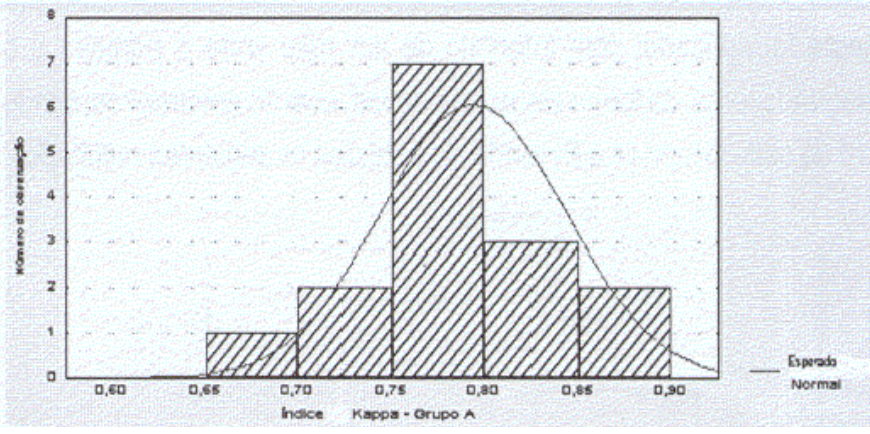

Figura 1 - Distribuição do Índice Kappa do Grupo A segundo o nível de concordância nas questões referentes à competência técnica em HIV/AIDS. Natal/RN, 1999

Verificamos que entre os índices Kappa do Grupo A, o menor foi 0,70 e o maior 0,87 , com média de 0,79 e desvio padrão de 0,05 . Esses resultados apontam para um bom nível de concordância entre os pesquisados no Grupo A.

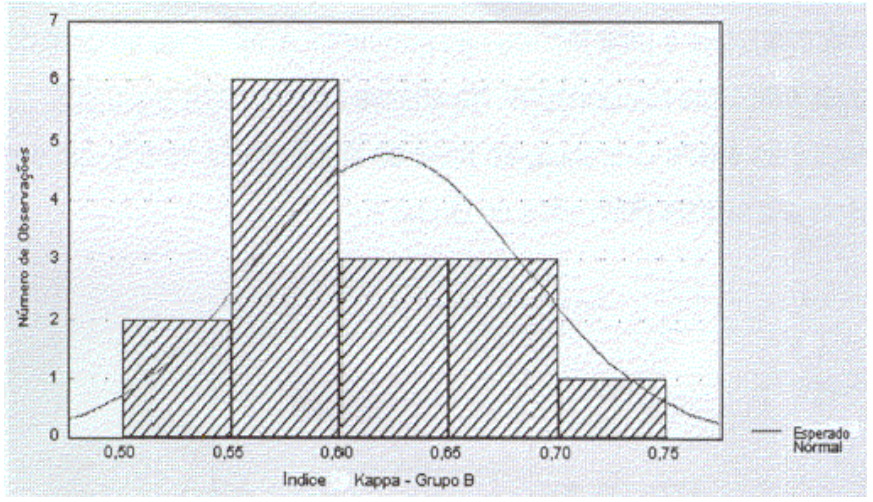

Figura 2 - Distribuição do Índice Kappa do Grupo B segundo o nível de concordância nas questões referentes à competência técnica em HIV/AIDS. Natal/RN, 1999

Observando a Figura 2, verificamos que dentre os índices Kappa do Grupo B, o menor foi 0,53 e o maior 0,73, com média de 0,60 e desvio padrão de 0,06 . Se levarmos em consideração, a média dos participantes do Grupo B, verificamos um índice Kappa regular entre os observadores deste grupo. Esses resultados apontam para uma diferença de concordância entre os Grupos A e B, ou seja, o Grupo A, obteve um nível de concordância superior ao grupo não treinado.

Para verificar a existência de diferença significativa entre os Grupos A e B, utilizamos a técnica Modelos Lineares Generalizados. Nessa análise encontramos diferença significativa, ao nível de significância de $5 \%$, entre os grupos de treinados e os não treinados em HIVIAIDS com chance de 1,045 em favor dos treinados.

Visando verificar a fidedignidade pelo método da estabilidade da parte II do instrumento, referente à competência técnica em HIVIAIDS, utilizamos a técnica do teste-reteste. Um novo 
teste foi aplicado, com intervalo de um mês entre o primeiro e 0 segundo teste, de forma aleatória simples, com 20 enfermeiros, sendo 10 de cada Grupo (A e B) tendo sido obtidos os seguintes resultados.

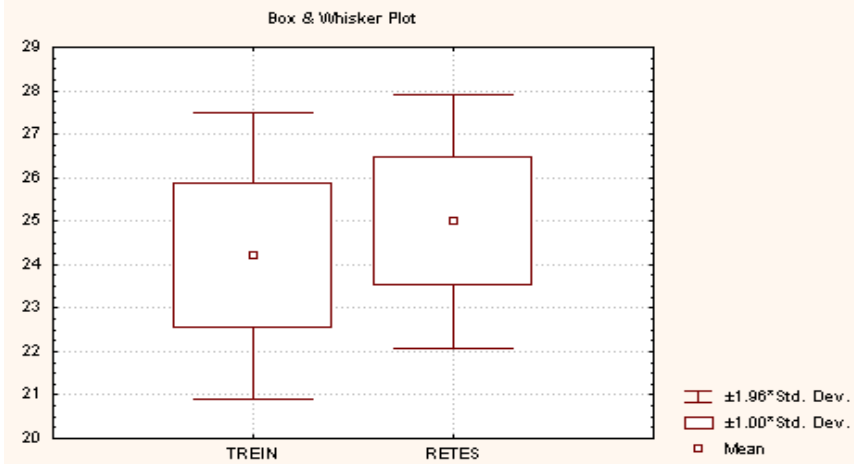

Figura 3 - Concordância entre o teste e o reteste do Grupo A. Natal/ RN, 1999

Para verificar o nível de estabilidade utilizamos a prova de Wilcoxon, que é uma técnica não paramétrica, aplicável ao caso de duas amostras relacionadas. $\mathrm{Na}$ análise encontramos $p>0,066$, ou seja, não existe diferença, ao nível de significância de $5 \%$, entre 0 teste e o reteste realizados no Grupo A.

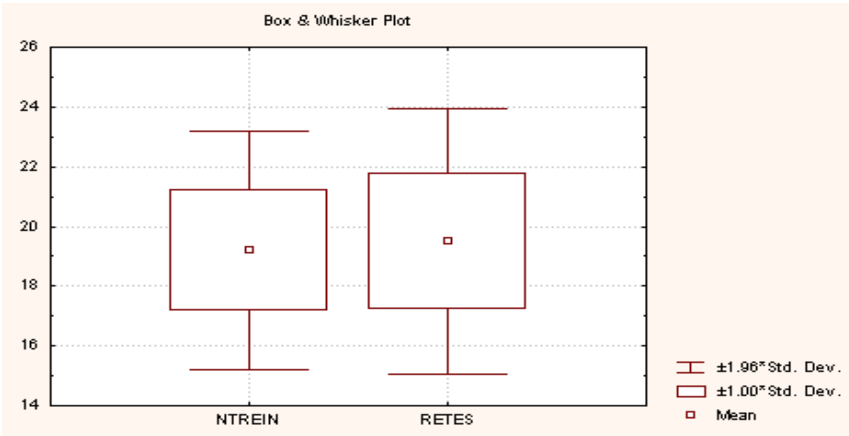

Figura 4 - Concordância entre o teste e o reteste do Grupo B. Natal/ RN, 1999

Ao realizarmos a análise, encontramos $p>0,441$, ou seja, não existe diferença significativa, ao nível de significância de $5 \%$, entre os testes realizados no Grupo B.

Através da técnica de Wilcoxon, não houve diferença significativa, ao nível de significância de $5 \%$, entre os testes realizados nos dois grupos. As médias dos escores obtidos nos dois testes foram, Grupo A ( $X_{1}=24,2$ e $\left.X_{2}=25\right)$ e Grupo $B\left(X_{1}=18,2\right.$ e $\left.X_{2}=18,7\right)$.

Desta forma, 0 conjunto das 30 questões relativas à competência técnica em HIVIAIDS contidas na parte II do instrumento, apresenta um nível de estabilidade e reprodutibilidade satisfatório, sendo portanto confiável.

Na Figura 5 podemos observar, como se comportaram as variações nas médias de escores nas sub-questões nos dois testes realizados, como também, a taxa de concordância entre eles.

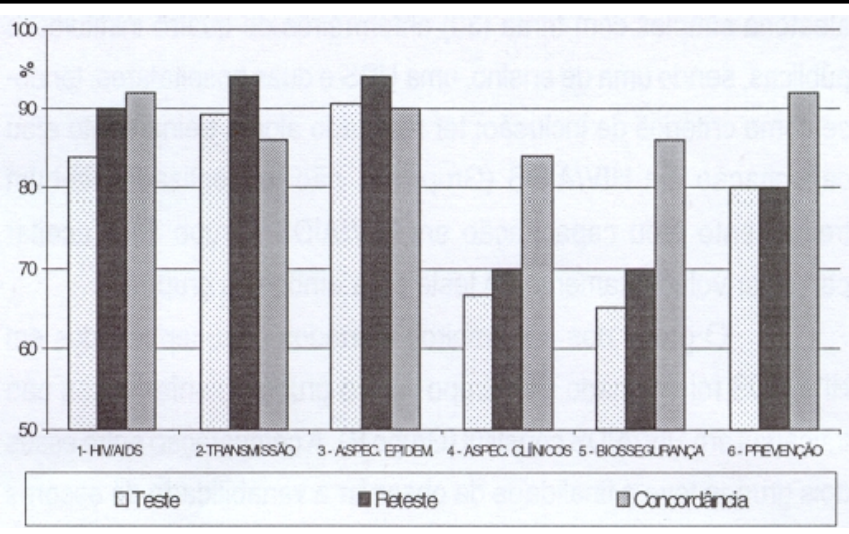

Figura 5 - Percentuais médios de acertos e concordância no teste e reteste das sub-questões sobre competência técnica do Grupo A. Natal/ RN, 1999

Ainda na Figura 5 observa-se homogeneidade satisfatória nas variações das sub-questões obtidas no teste e no reteste realizados no Grupo A. Verifica-se nos escores obtidos, um discreto aumento nas médias dos escores das sub-questões, com exceção da sub-questão relativa à prevenção, por ocasião do reteste e média de concordância entre os testes de $88,3 \%$, com intervalo de confiança de 70 a $100 \%$.

$\mathrm{Na}$ Figura 6, observa-se variação nas médias dos escores obtidos na sub-questão relativa ao vírus HIV (54,8\% e 70\%) durante os testes realizados no Grupo B, com discreto aumento nas médias dos escores nas demais sub-questões. A média de concordância entre os testes foi de $80,3 \%$, com intervalo de confiança de 63 a $98 \%$.

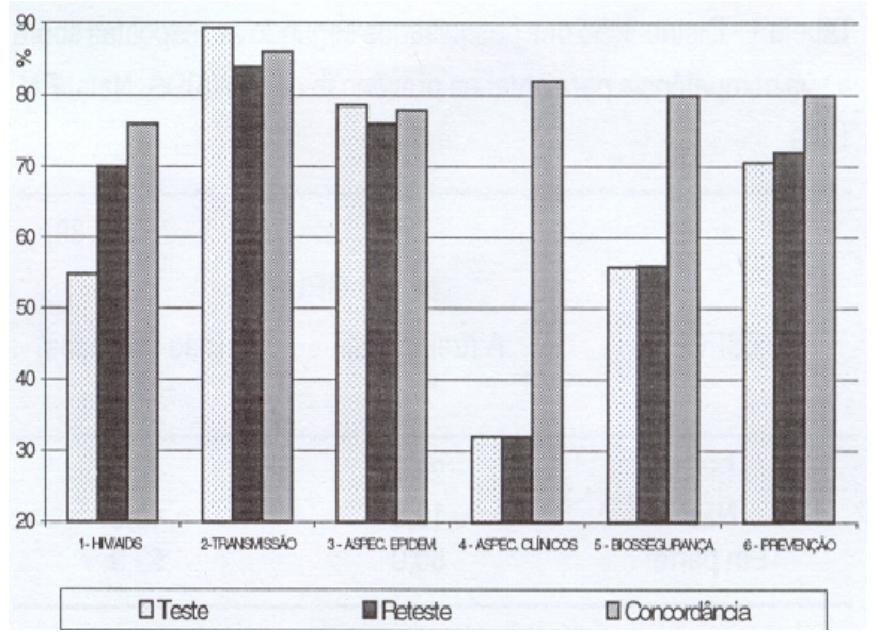

Figura 6 - Percentuais médios de acertos e concordância no teste das sub-questões sobre competência técnica do grupo B. Natal/RN, 1999

Os resultados nas variações das médias, obtidas nas subquestões, apontam para uma boa concordância entre os aspectos abordados nos sub-ítens, demonstrando nível satisfatório de homogeneidade do instrumento que segue. 
INSTRUMENTO SOBRE COMPETÊNCIA TÉCNICA EM HIVI AIDS E SUA PREVENÇÃO

\section{I - CARACTERIZAÇÃO DOS SUJEITOS}

\section{Dados pessoais:}

1.1-Gênero: ( ) M ( ) F

1.2- Faixa etária (anos): ( ) menos de 20 ( ) 20 a 25 ( ) 26 a 30
( ) 31 a 35
( ) 36 a 40 ( ) 41 a 45

( ) mais de 45

2. Local de Trabalho:

2.1- Distrito Sanitário: ( ) Norte ( ) Sul ( ) Leste ( ) Oeste

( ) Nível Central

2.2- Nome da Unidade de Saúde:

2.3- Tempo de serviço (em anos) na SMS: e na UBS

\section{Formação/Qualificação profissional:}

3.1- Tempo de conclusão do Curso de Graduação em Enfermagem (em anos):

3.2- Curso de Licenciatura em Enfermagem: ( ) Sim, há quantos anos: ( ) Não

3.3- Curso de Especialização: ( ) Sim, há quantos anos:

e em que área: __ C $\mathrm{CH}$ :__ ( ) Não

3.4- Curso de Mestrado: ( ) Sim, há quantos anos: e em

que área: $\mathrm{CH}$ : ( ) Não

3.5- Marque no quadro abaixo qual(is) o(s) treinamento(s) e/ou capacitação que você realizou em DST/HIVIAIDS

\begin{tabular}{|l|l|l|l|}
\hline TREINAMENTO/CAPACITA/CÃO & CH & ANO & N. VEZES \\
\hline Aconselhamento em DST/HIVIAIDS & & & \\
AIDS em obstetrícia & & & \\
Capacitação técnica para enfermeiros & & & \\
em HIVIAIDS & & & \\
DST e AIDS & & & \\
Manejo clínico de gestantes & & & \\
Multiplicador na área de adolescentes & & & \\
Outros treinamentos: & & & \\
\hline
\end{tabular}

3.6-Você gostaria de fazer algum treinamento ou curso relacionado a temática do HIVIAIDS?

( ) Não ( ) Sim, Qual(is):

\section{II - QUESTÕES SOBRE A COMPETÊNCIA TÉCNICA EM HIVI} AIDS E SUA PREVENÇÃO

1- Você se considera competente tecnicamente para atuar na prevenção do HIVIAIDS nas UBS?

( ) Sim ( ) Em parte ( ) Não, Justifique:

2- Os itens a seguir referem-se aos aspectos relativos ao HIV/ AIDS e sua prevenção. Responda todas as questões, segundo seu conhecimento sobre as mesmas, colocando nos parênteses, $V$ se você considera a afirmativa verdadeira e $F$ se você considera falsa.

2.1- No que se refere ao Vírus HIV causador da AIDS podemos afirmar que:

$\left(V^{*}\right)$ A produção de partículas virais é contínua em todos os estágios da infecção. $\left(100 \%{ }^{* *}\right)$

(V) Existem dois tipos de HIV, denominados retrovírus HIV-1 e HIV-2. (100\%)

(F) $O$ tempo entre a contaminação pelo vírus HIV-1 e a manifestação da AIDS é até 2 anos. (100\%)
(V) O HIV-1 é o principal vírus causador da AIDS no Brasil. (100\%) (F) $O$ vírus HIV surgiu como consequêencia dos comportamentos de riscos dos homossexuais, prostitutas e usuários de drogas injetáveis. (100\%)

2.2- Quanto as fontes de infecção e formas de transmissão do HIV é correto afirmar que:

(V) $O$ sangue, sêmen, fluidos vaginais e o leite materno são fontes de infeç̧ão do HIV. (100\%)

(V) A principal forma de transmissão do HIV se dá hoje através de relações sexuais, com incidência maior em heterossexuais. (100\%) (V) Na transmissão vertical, o bebê de uma mãe portadora do HIV pode nascer infectado e assintomático. (100\%)

(F) $O$ sexo anal e vaginal sem proteção são de médio risco para a transmissão do HIV. (100\%)

(V) As doenças sexualmente transmissíveis ulcerativas aumentam numerosas vezes a possibilidade de transmissão do HIV. (80\%) 2.3 - Com relação aos aspectos epidemiológicos do HIVIAIDS é correto afirmar que:

(V) $O$ grupo etário mais atingido pela AIDS no Brasil tem sido o de 20 a 39 anos. (100\%)

(V) A feminilização, heterossexualização, a juvenização, interiorização e pauperização são características epidemiológicas da AIDS no Brasil. (100\%)

(V) $\mathrm{O}$ aumento da incidência de mulheres e crianças infectadas pelo HIV são indícios da transmissão heterossexual. (100\%)

(F) Os principais responsáveis pela disseminação da AIDS no Brasil atualmente são os homossexuais. (100\%)

(V) O Brasil situa-se entre os quatro países do mundo com maior prevalência de casos notificados de AIDS. (100\%)

2.4- No tocante aos aspectos clínicos e laboratoriais do HIVIAIDS é correto afirmar:

(F) A célula alvo do HIV é o eritrócito T4. (100\%)

(V) A janela imunológica é o tempo compreendido entre aquisição da infecção pelo HIV e a soroconversão. (100\%)

(F) Quando a Aids se manifesta na fase aguda o resultado é sempre fatal. (80\%)

(F) O Elisa e o Western-Blot utilizadas na rotina no diagnóstico do HIV, são exames baseados na detecção de antígenos contra 0 vírus HIV. (80\%)

(F) $O$ teste Elisa é o mais utilizado para o diagnóstico da positividade do HIV por ser mais sensível e específico. (80\%)

2.5- No tocante as normas de biossegurança em relação ao HIV/ AIDS é correto afirmar:

(V) Sempre que houver probabilidade de exposição a sangue ou fluidos orgânicos é recomendado o uso de equipamentos de proteção individual (EPI). (100\%)

(V) Os líquidos, como sangue e fluidos orgânicos, podem ser descartados numa fossa sanitária (química). $(80 \%)$

(V) 0 glutaraldeído e o hipoclorito de sódio a $1 \%$ possuem ação desinfectante após 30 minutos de exposição nos artigos contaminados. (80\%)

(F) Os agentes físicos, como autoclave e estufa, necessitam de um tempo de exposição de 30 minutos a uma temperatura de $120^{\circ} \mathrm{C}$ para esterilização dos artigos contaminados. (80\%)

(F) As medidas de biossegurança devem ser adotadas, especificamente, em procedimento invasivo ou contato com fluidos corporais de indivíduos soropositivos para o HIV. (100\%) 
2.6- Quanto ao aconselhamento pré-teste e prevençẫo do HIV/ AIDS é correto afirmar que:

(F) 0 aconselhamento pré-teste destina-se aos indivíduos com comportamentos de risco para o HIV, como os profissionais do sexo, homossexuais e usuários de drogas injetáveis. ( $80 \%$ ) $(\mathrm{V})$ Ter relaçã̃o sexual com preservativo e reduzir o número de parceiros é uma forma de sexo seguro. ( $80 \%)$

(V) A eficiência dos preservativos na prevençẫo do HIV depende da qualidade do seu uso. (100\%)

\section{CONSIDERAÇÕES FINAIS}

Os resultados alcançados neste estudo através da validação aparente e de conteúdo do instrumento, como também, da análise psicométrica mostraram nível satisfatório de validação e estabilidade, homogeneidade e reprodutibilidade do instrumento.

\section{REFERÊNCIAS BIBLIOGRÁFICAS}

1. Torres GV. Atividades educativas na prevenção da AIDS em uma rede básica municipal de saúde: participação do enfermeiro. [dissertação]. João Pessoa/PB: Universidade Federal da Paraíba; 1997.

2. Moriya TM. Escala de atitudes frente à AIDS: uma análise psicométrica. [tese]. Ribeirão Preto/SP: Escola de Enfermagem de Ribeirão Preto/USP; 1992.

3. Gir E. Práticas sexuais e a infecção pelo HIV: um estudo sobre crenças entre universitários de Ribeirão Preto/SP. [tese]. Ribeirão Preto/SP: Escola de Enfermagem de Ribeirão Preto/USP; 1994.

4. Llobera Serentill A, Casabella Abril B, Perez Sanchez J, Carrillo Munoz R, Via Vidal M, Garcia Ortega D. Elaboracion de una prueba para evaluar conocimientos y actitudes sobre la infeccion por el virus de la inmunodeficiencia humana en profesionales de atencion primaria de salud. Aten Primaria 1996 Mayo; 17(9):564-9.
(V) A prevençẫo e controle do HIV/AIDS é de responsabilidade individual e coletiva. (100\%)

(F) A reduçẫo da disseminaçã̃o do $\mathrm{HIV}$ depende da proibiçã̃o do liberalismo sexual. (100\%)

Obs:

* Gabarito para correçẫo das questỗes sobre competência técnica em HIVIAIDS.

** Percentuais de concordância entre os juízes, na avaliaçẫo do poder discriminativo de cada item.

Desta forma, reconhecendo a importância de refinamento quanto a outras possíveis análises como sensibilidade e especificidade, 0 instrumento elaborado pode ser considerado satisfatório, adequado e útil na mensuração da competência técnica na prevenção do HIV/ AIDS.

5. Ministério da Saúde (BR). Catálogo de ações, produtos e serviços em DST/AIDS no local de trabalho. Brasília (DF): Ministério da Saúde; 1997a.

6. Ministério da Saúde (BR). Manual de orientação básica para equipe de enfermagem: prevenção do HIV e assistência a pessoas portadoras do HIV e de AIDS. Brasília (DF): Ministério da Saúde;1997b.

7. Ministério da Saúde (BR). Aconselhamento em DST, HIV e AIDS: diretrizes e procedimentos básicos. 2. ed. Brasília (DF): Ministério da Saúde; 1998.

8. Vianna HM. Testes em educação. São Paulo (SP): IBRASA; 1982.

9.Pereira MG. Aferição dos eventos. In: Pereira MG. Epidemiologia: teoria e prática. 2. ed. Rio Janeiro (RJ): Guanabara Koogan; 1999. p. 358-76. 